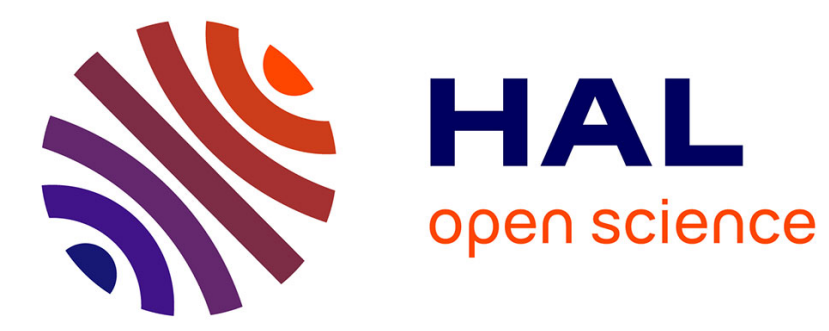

\title{
Études de magnétisme réalisées avec des neutrons
}

\author{
M. Ericson, P.-G. de Gennes, A. Herpin, B. Jacrot, P. Meriel
}

\section{To cite this version:}

M. Ericson, P.-G. de Gennes, A. Herpin, B. Jacrot, P. Meriel. Études de magnétisme réalisées avec des neutrons. J. Phys. Radium, 1958, 19 (7), pp.617-623. 10.1051/jphysrad:01958001907061700 . jpa-00235904

\section{HAL Id: jpa-00235904 https://hal.science/jpa-00235904}

Submitted on 1 Jan 1958

HAL is a multi-disciplinary open access archive for the deposit and dissemination of scientific research documents, whether they are published or not. The documents may come from teaching and research institutions in France or abroad, or from public or private research centers.
L'archive ouverte pluridisciplinaire HAL, est destinée au dépôt et à la diffusion de documents scientifiques de niveau recherche, publiés ou non, émanant des établissements d'enseignement et de recherche français ou étrangers, des laboratoires publics ou privés. 


\title{
LE JOURNAL DE PHYSIQUE
}

ET

\author{
LE RADIUM
}

\section{ETUDES DE MAGNÉTISME RÉALISÉES AVEC DES NEUTRONS}

\author{
Par M. ERICŚON, P. G. DE GENNES, A. HERPIN, B. JACROT et P. MÉRIEL, \\ C. E. N., Saclay.
}

\begin{abstract}
Résumé. - On décrit deux expériences de diffusion des neutrons par des substances magnétiques. Dans la première, on étudie au moyen d'un spectromètre à ́cristal la structure antiferromagnétique de $\mathrm{FeCl}_{2}$ et l'action d'un champ magnétique extérieur sur la disposition des moments magnétiques.

La seconde, effectuée avec un spectromètre à temps de vol, a pour objet la diffusion critique au voisinage du point de Curie du fer. Elle permet de déterminer les deux paramètres caractérisant les fluctuations d'aimantation, la partie des corrélations et leur constante de diffusion.
\end{abstract}

Abstract. - This paper describes two series of experiments on neutron scattering by magnetic media. Series A gives the antiferromagnetic structure of $\mathrm{FeCl}_{2}$, and the effect of a magnetic field on the spin orientations as measured with a crystal spectrometer. In series $\mathrm{B}$, the critical magnetic scattering of. iron near the Curie Point has been investigated with a time of flight spectrometer. As a result one gets both the range of the spin correlations and the scattering constant which is related to their decay in time.

1. Les neutrons qui sortent d'un canal de pile sont en équilibre thermique avec la matière dans laquelle ils se sont ralentis. Leur répartition spectrale est donc maxwellienne et la longueur d'onde correspondant au maximum du flux est de $1,8 \AA$. Cette longueur d'onde, du même ordre de grandeur que celle des rayons $X$ utilisés pour l'étude de structures cristallines, permet de faire des études de cristallographie en vue de déterminer la position des atomes dans la maille cristalline. De plus les neutrons possèdent un moment magnétique égal à 1,91 magnéton de Bohr nucléaire, qui peut interagir avec les moments magnétiques atomiques. Par étude de la diffraction des neutrons, nous pourrons donc localiser les porteurs de moments magnétiquess dans la maille cristalline. C'est là un des avantages principaux des neutrons sur les rayons $\mathrm{X}$ et qui justifie leur emploi, malgré les difficultés rencontrées (rareté des neutrons - intensité des flux relativement faible - impossibilité d'une détection sur film).

Dans les conditions usuelles, c'est-à-dire pour des neutrons non polarisés et des noyaux non orientés, l'intensité neutronique diffusée est la somme de l'intensité diffusée par les noyaux et de l'intensité provenant de la diffusion magnétique, sans qu'il y ait entre elles d'interférences :

$$
I=I_{\mathrm{N}}+I_{\mathbf{M}}
$$

L'intensité « nucléaire » diffusée par un cristal a pour expression

$$
I_{\mathrm{N}}=A\left|\underset{j}{\sum_{j}} b_{j} \exp \left(i \mathbf{K} \mathbf{R}_{j}\right)\right|^{2}
$$

La sommation est étendue à tous les atomes, repérés par le vecteur $\mathbf{R}_{\boldsymbol{j}}$. $A$ est une constante et $\mathbf{K}$ le vecteur de diffusion

$$
\mathbf{K}=2 \pi\left(\frac{\mathbf{s}_{\mathbf{0}}}{\lambda}-\frac{\mathbf{s}}{\lambda}\right)=K . \mathbf{e}
$$

où $\lambda_{0}$ est la longueur d'onde des neutrons-incidents dont la direction de propagation est repérée par le vecteur unitaire $\mathbf{s}_{\mathbf{0}} ; \lambda$ et $\mathbf{s}$ se rapportient aux neutrons diffusés.

$b_{j}$ est l'amplitude de diffusion cohérente du noyau $j$. C'est une constante, ayant les dimensions d'une longueur, telle que la section efficace de diffusion cohérente soit

$$
\sigma_{j}=4 \pi b j_{2} .
$$




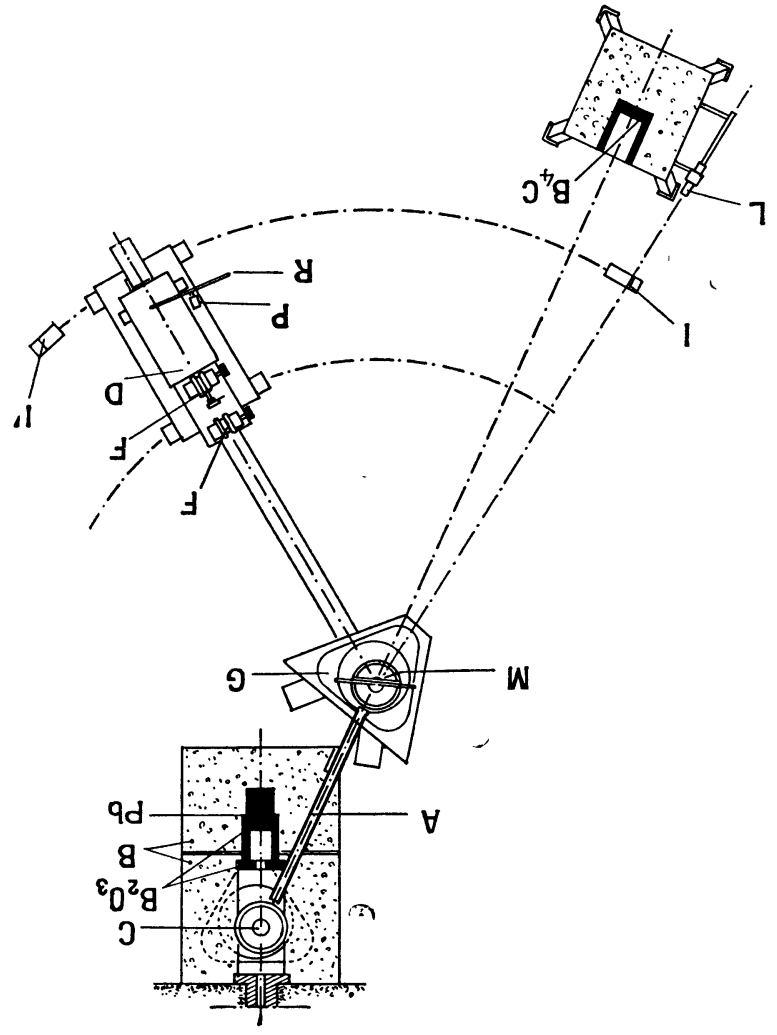

FIG. 1.
L'intensité " magnétique » diffusée a une expression analogue à (2), en remplaçant les amplitudes de diffusion nucléaires par les amplitudes de diffusion ( magnétique » $b_{j}^{(m)}$ qui dépendent :

a) de la longueur du vecteur de diffusion $\mathbf{K}$, ce qui traduit les dimensions finies des couches électroniques responsables de l'existence des moments magnétiques atomiques, d'où un facteur de forme $f_{j}(K)$;

b) de la direction du moment magnétique $\mathbf{M}_{j}$ de l'atome $j$ par rapport au vecteur de diffusion $\mathbf{K}$. Pour un neutron de spin $\mathbf{s}$, la longueur de diffusion magnétique de l'atome $j$ est

$$
b^{(m)}=b_{0} f_{j}(K)\left[\left(\mathbf{e} \mathbf{M}_{j}\right) \mathbf{e}-\mathbf{M}_{j}\right] . \mathbf{s}
$$

où $b_{0}$ est une constante ,égale à $\frac{e^{2} \gamma}{m c^{2}}(\gamma=$ rapport gyromagnétique du neutron). Dans le cas où tous les moments magnétiques sont parallèles ou antiparallèles, l'intensité magnétique est proportionnelle au carré du sinus de l'angle que font les moments magnétiques avec le vecteur de diffusion, comme on le voit facilement sur la formule (5). L'étude de l'intensité " magnétique " pourra donc nous permettre de déterminer la position et la direction des moments magnétiques atomiques.

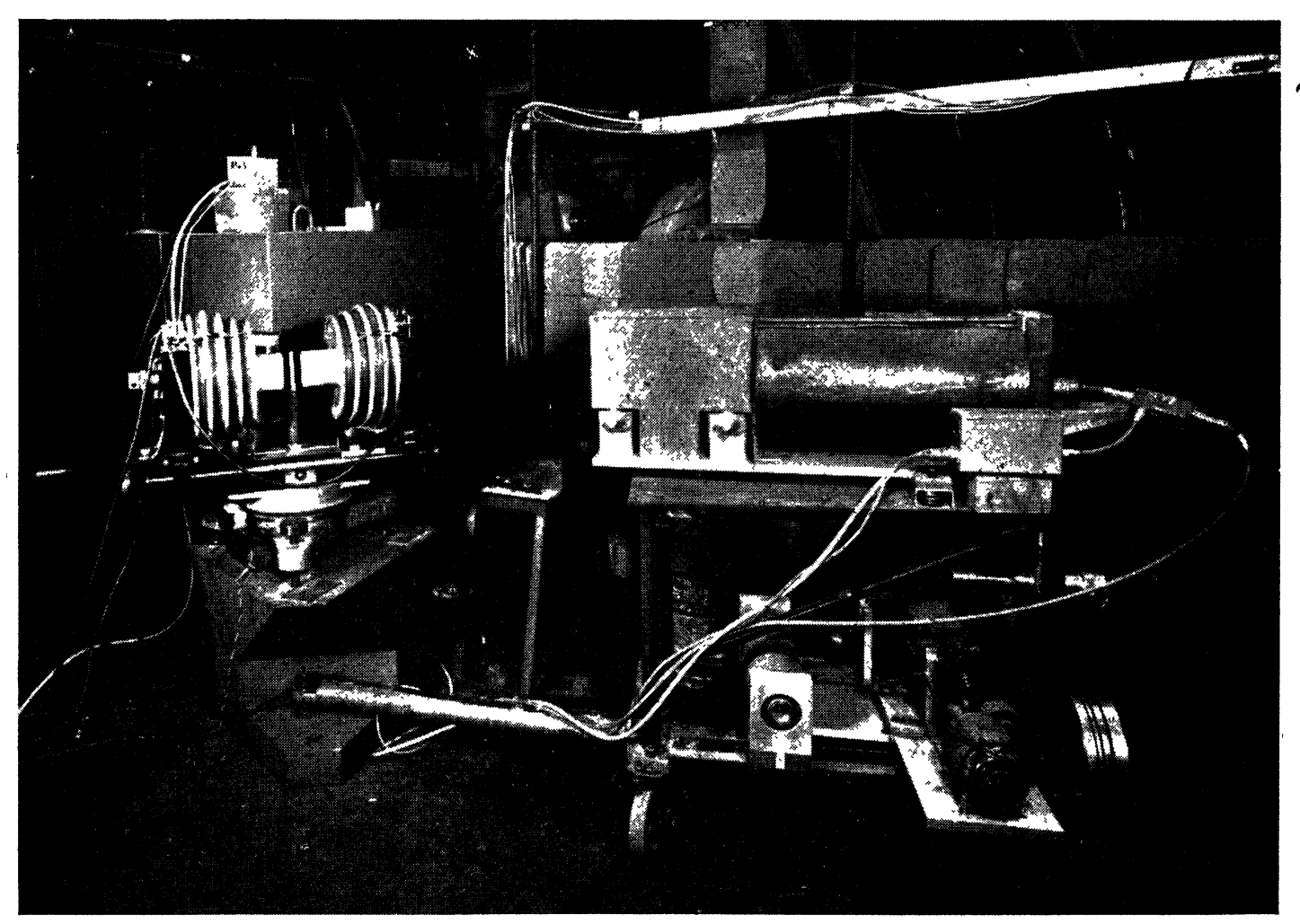

FIG. 2. 
2. Techniques expérimentales. - Les techniques utilisées dans la physique des neutrons n'étant pas encore très classiques en France, nous nous permettrons de rappeler très brièvement les dispositifs expérimentaux utilisés. Le but à atteindre est : 1) Isoler, dans tout le spectre des neutrons sortant d'un canal de pile, un faisceau de neutrons monocinétiques (monochromatiques) de longueur d'onde $\lambda_{0}$ et d'énergie $E_{0} .2$ ) Étudier : a) la répartition des neutrons diffusés en fonction de l'angle de diffusion soit $n(\theta)$ ce nombre ; b) le spectre énergétique des neutrons diffusés dans une direction donnée, $n(\theta, E)$.

A. Spectromètre a cristal. - Le plus simple, pour obtenir des neutrons monocinétiques est d'utiliser un monochromateur à cristal, analogue, à l'échelle près, à ceux que l'on emploie dans le cas des rayons $X$. Le faisceau de neutrons, collimaté dans le canal de la pile, tombe sur un monocristal dont une famille de plans réticulaires distants de $d$ fait un angle $\theta$ avec l'axe du faisceau. Les neutrons réfléchis sélectivement ont une longueur d'onde $\lambda_{0}=2 d \sin \theta$. Il suffit d'éliminer tous les autres neutrons, ce qu'on réalise au moyen d'une lourde protection percée d'un canal. Dans les expériences de diffraction cristalline, les neutrons diffractés ont en majeure partie la même énergie que les neutrons incidents (diffusion élastique) et pour les étudier, il suffit d'explorer les différents angles de diffusion au moyen d'un détecteur (généralement un comptêur à $\mathrm{BF}_{3}$ enrichi en isotope ${ }^{10} \mathrm{~B}$ ). Les figures 1 et 2 montrent un plan schématique et une vue d'ensemble du spectromètre utilisé à Saclay [1]

Si on veut déterminer la répartition énergétique des neutrons diffusés par l'échantillon dans une direction donnée, il suffit d'analyser le flux diffusé au moyen d'un second spectromètre à cristal, comme le montre la figure 3. Mais un spectromètre à cristal est un appareil dont la luminosité est très faible, et ce dispositif ne peut être utilisé que si le flux de neutrons sortant de la pile est suffisamment élevé, comme c'est le cas pour la pile canadienne de Chalk-River [2].

B. SÉlecteur a temps de vol. - Lorsqu'on ne dispose que d'une pile de flux moyen, il est nécessaire de recourir à un dispositif expérimental différent, le spectromètre à temps de vol, dont le principe est assez simple, mais qui nécessite un appareillage mécanique et électronique très complexe.

Considérons un neutron diffusé à l'instant $t=0$, et dont la vitesse est $v$. Il atteindra le détecteur, placé dans une direction donnée, à une distance $L$, de l'échantillon à un instant $t=L / v$. Le faisceau de neutrons est haché par une roue à bras haute $L_{1}$ percée d'une fente radiale tournant à une vitesse angulaire $\omega$, de l'ordre de 6000 tours/minute.
L'instant origine est déterminé par le passage de la fente devant le faisceau. Ce passage déclenche un ensemble de numérateurs retardés. Ces numérateurs sont au nombre de $N(N=100$ dans le spectromètre

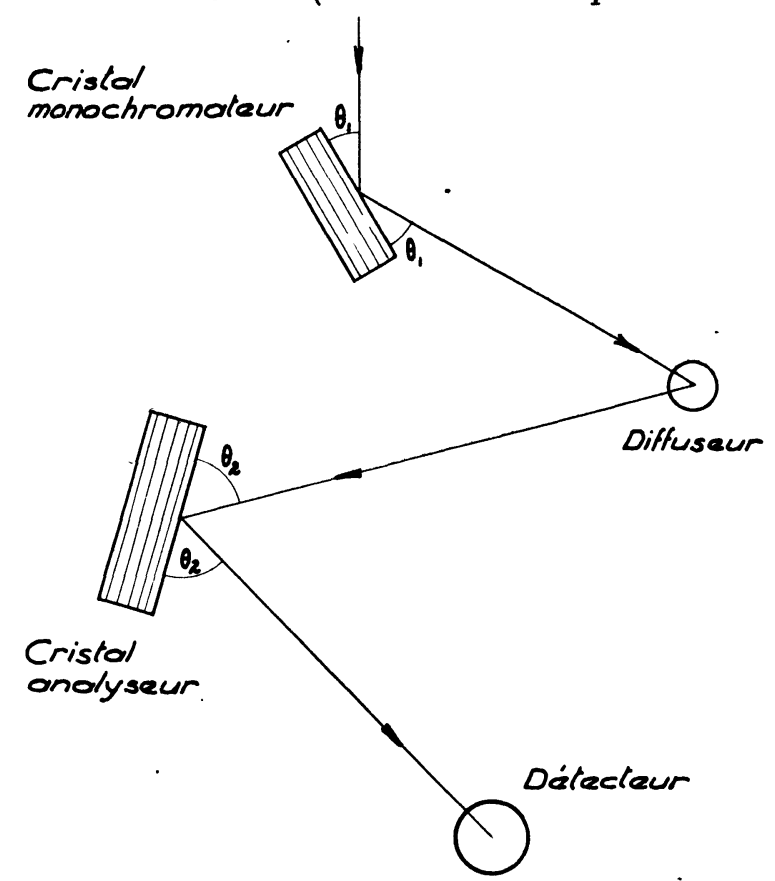

FIG. 3.

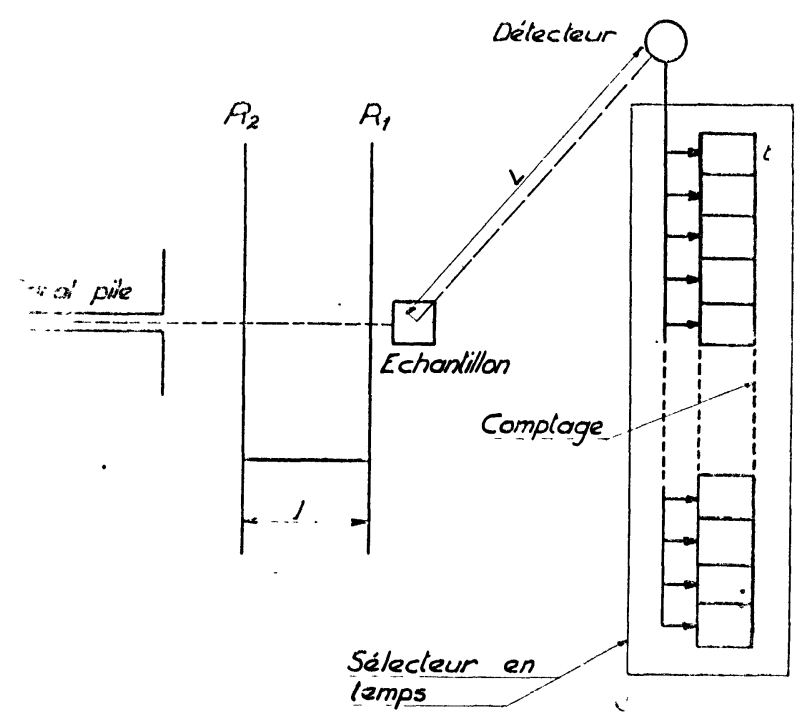

FIG. 4.

utilisé à Saclay) et le numérateur $\mathrm{n}$ totalise les neutrons qui ont atteint le détecteur à un temps $t$ compris entre $(n-1) \tau$ et $n \tau$; c'est-à-dire les neutrons dont la vitesse est comprise entr $L / n \tau$ et $L(n-1) \tau$. $\tau$ est une constante de temps de l'ordre de $10 \mu \mathrm{s}$. Grâce à ce dispositif, le spectre des neutrons diffusés est directement enregistré dans les $N$ numérateurs.

Pour monochromatiser les neutrons, on utilise 
une roue $L_{2}$, analogue à la roue $L_{1}$, placée avant $L_{1}$ sur le trajet des neutrons sortant de la pile, la distance séparant les deux roues étant $l$. La fente de $L_{2}$ est décalée d'un angle $\alpha$ par rapport à celle de $L_{1}$. Les neutrons de vitesse $v_{0}=l \omega / \alpha$ passent à travers les fentes des deux roues; les neutrons plus rapides tombent en avant de la fente de $L_{1}$, les neutrons plus lents tombent en arrière. La figure 5 représente la forme de la raie monochromatisée ainsi obtenue. Sa largeur est due à la largeur des fentes et au manque de parallélisme du faisceau de neutrons collimaté à l'intérieur du canal de la pile.

Pour augmenter la luminosité de l'appareil,

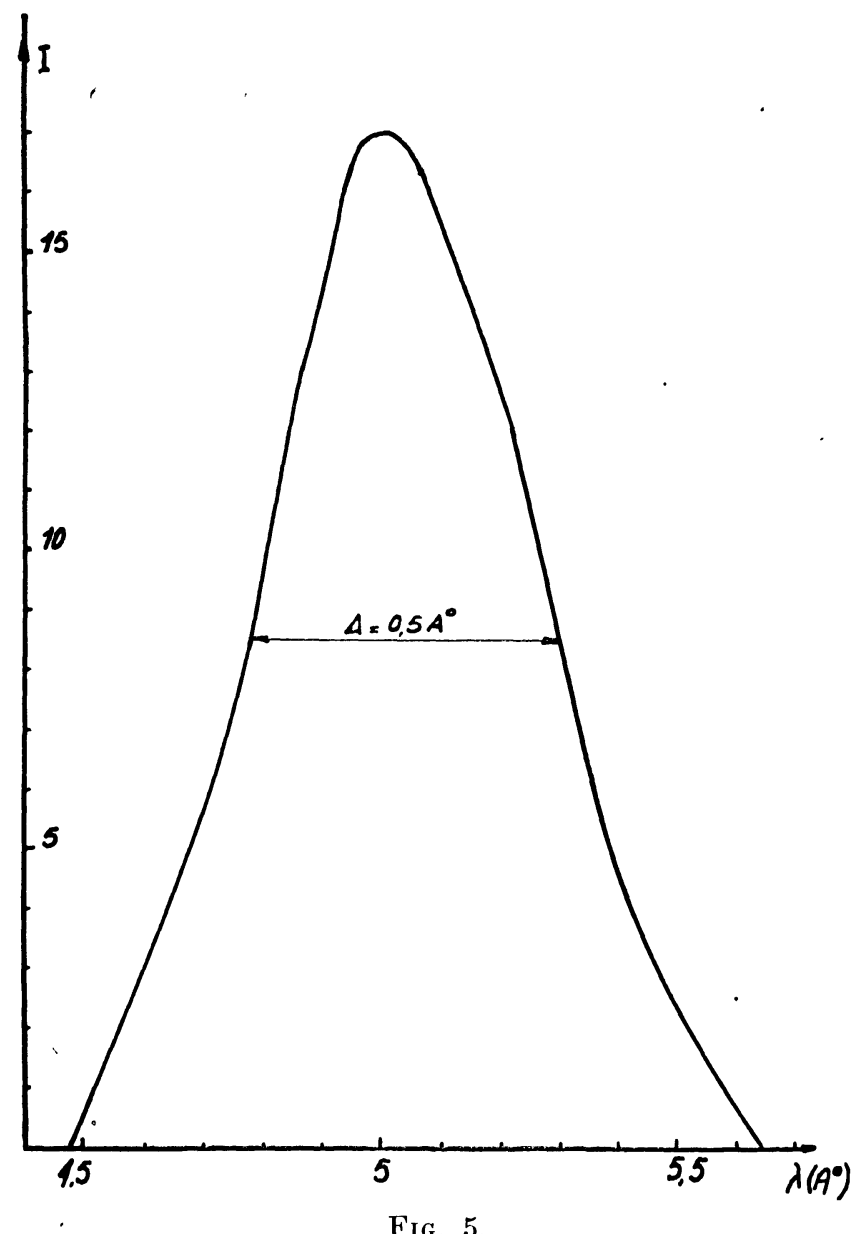

chaque roue porte plusieurs fentes. Une vue d'ensemble du sélecteur mécanique installé devant la pile de Saclay a été publiée dans ce journal [3].

3. Structure antiferromagnétique du chlorure ferreux anhydre. - Nous avons entrepris des expériences sur le chlorure ferreux anhydre en vue de mettre en évidence l'action du champ magnétique sur la disposition des moments magnétiques. En effet, les mesures magnétiques ont montré que $\mathrm{FeCl}_{2}$ était à basse température un antiferromagnétique d'un type particulier, appelé métamagnétique : son couplage antiferromagnétique est assez faible pour que, sous l'action d'un champ magnétique modéré, ses moments magnétiques soient parallèles.

A. Structure magnétique de $\mathrm{FeCl}_{2} \cdot-\mathrm{FeCl}_{2}$ cristallise dans le système rhomboédrique. Sa struc-

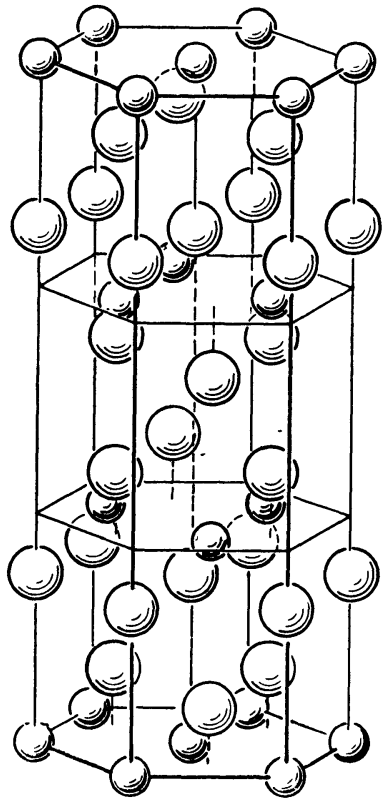

Fig. 6.
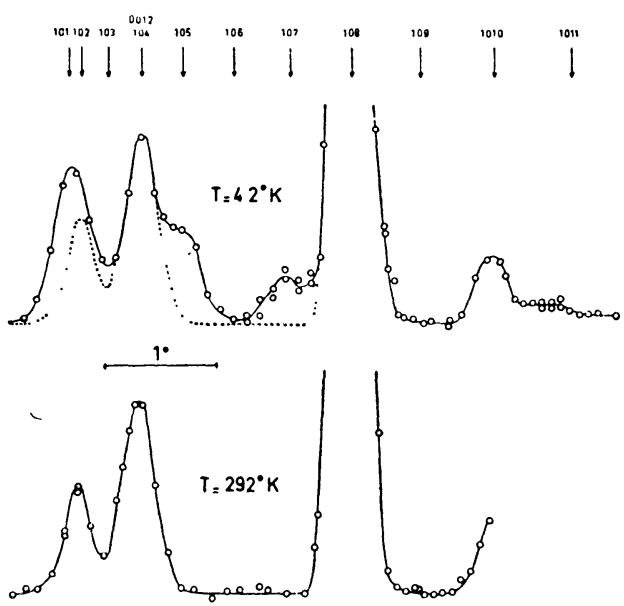

Spectres de diffraction de neutrons de $\mathrm{FeCl}$.

Fig. 7.

ture est représentée sur la figure 6 avec la maille hexagonale correspondante. C'est un empilement de feuillets d'atomes de fer, séparés par deux feuillets d'atomes de chlorure, chaque maille cristallographique contenant trois feuillets de fer. La figure 7 montre les spectres de diffraction obtenus 
à la température ordinaire et à la température de l'hélium liquide. On voit qu'il apparaît à basse température quatre raies supplémentaires qui traduisent l'existence d'une surstructure magnétique. On interprète ces raies en admettant que les atomes de fer d'un même feuillet ont leurs moments parallèles, les moments de deux feuillets voisins étant antiparallèles. Il en résulte que la maille " magnétique " a un paramètre $c$ double celui de la maille cristallographique. Dans cette nouvelle maille, les raies de surstructure peuvent s'indexer $(1,0, l)$ où $l=1,5,7,11$. En outre l'absence de la raie $(0,0,3)$ impose aux moments atomiques une direction parallèle à l'axe $c$. Ces résultats sont en accord avec ceux obtenus par Wilkinson et Cable [5]. La figure 8 montre l'accord entre les intensités mesurées et calculées à partir de ce modèle, ces intensités étant normalisées à leur somme.

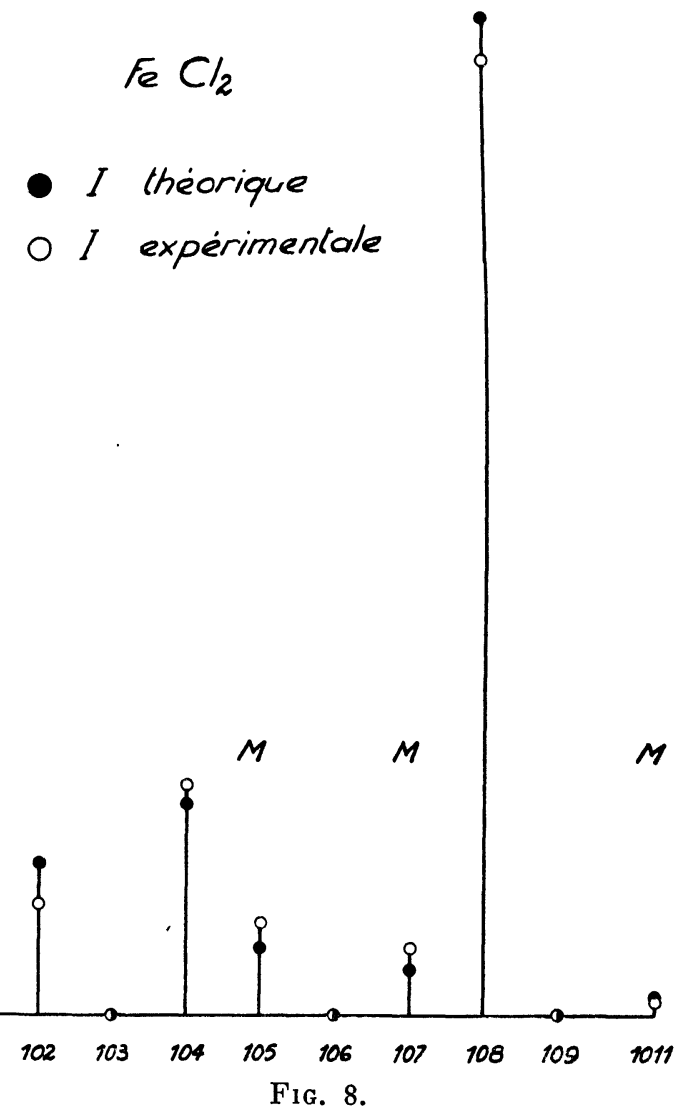

B. Étude DE L'ACTION D'Un CHAMP MaGNÉTIQUE. - La direction du champ magnétique reste parallèle au vecteur de diffusion. Pour tous les cristallites qui participent à une réflexion sélective $(h, k, l)$, le vecteur de diffusion $\mathbf{K}$ coïncide avec le nœud $(h, k, l)$ du réseau réciproque. Tous ces cristallites ont donc même disposition cristallographique par rapport au champ magnétique. Si, de plus le cristal est uniaxe et si la dịection d'anti- ferromagnétisme $\Delta$ est l'axe $c$, pour tous ces cristallites $\Delta$ fait le même angle $\varphi$ avec le champ magnétique. Dans un cristal uniaxe on peut, au moins en première approximation, admettre que lorsqu'on applique un champ magnétique $\mathbf{H}$ les vecteurs aimantation des deux sous-réseaux $\mathbf{M}_{1}$ et $\mathbf{M}_{\mathbf{2}}$ primitivement antiparallèles dans la direction de $\Delta$ restent dans le plan formé par la direction d'antiferromagnétisme et le champ magnétique $\mathbf{H}$. Dans ces conditions les vecteurs $\mathbf{M}_{1}$ et $\mathbf{M}_{2}$ des divers cristallites contribuant à une même raie $(h, k, l)$ sont parallèles entre eux. Donc l'étude de la variation de l'intensité d'une raie, en fonction $d u$ champ est équivalente, aux interactions magnétiques entre cristallites près, à une étude sur monocristal. Les mesures magnétiques effectuées sur une poudre donnent au contraire des résultats qui sont une moyenne plus difficilement interprétable.

Dans un cristal métamagnétique, les aimantations $\mathbf{M}_{1}$ et $\mathbf{M}_{2}$ des deux sous-réseaux seront parallèles lorsque le champ magnétique sera supé-. rieur à un certain champ limite $H_{\mathbf{L}}(\varphi)$. L'intensité d'une raie magnétique est alors nulle. Mais la variation d'intensité en fonction du champ diffère suivant la valeur de l'angle $\varphi$. Cette intensité est en effet proportionnelle à

$$
\left(\sin \alpha_{1}-\sin \alpha_{2}\right)^{2}
$$

où $\alpha_{1}$ et $\alpha_{2}$ désignent les angles que font, avec la direction du champ les moments $\mathbf{M}_{1}$ et $\mathbf{M}_{2}$ des deux sous-réseaux (cf. fig. 9). Or la variation des

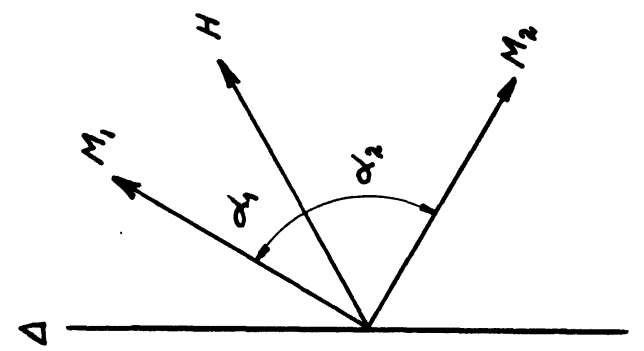

FIG. 9.

angles $\alpha_{1}$ et $\alpha_{2}$ en fonction du champ dépend de l'angle $\varphi$. Une étude théorique élémentaire montre que le champ limite $H_{\mathrm{L}}(\varphi)$ est d'autant plus élevé que l'angle $\varphi$ est plus voisin de $90^{\circ}$. Comme le champ magnétique dont nous disposions ne pouvait dépasser $15000 \mathrm{Oe}$, nous avions intérêt à étudier des raies magnétiques correspondant à des angles $\varphi$ petits. Mais l'intensité d'une telle raie, proportionnelle à $\sin ^{2} \varphi$, est faible. Nous avons adopté une solution intermédiaire, et nous avons étudié la variation de l'intensité de la raie $(1,0,7)$ en fonction du champ, pour laquelle l'angle $\varphi$ est égal à $57^{\circ}$. La figure 10 représente le résultat de cette étude. La courbe en tirets représente la variation calculée en supposant, comme l'a fait L. Néel, que l'anisotropie peut être représentée au moyen d'une seule constante $K$ et que $K / n M^{2}=0,25$, où $n$ est 
la constante de champ moléculaire et $M$ l'aimantation. Le champ critique a été ajusté pour obtenir e meilleur accord entre la courbe et les points expérimentaux. On trouve $H_{\mathrm{c}}=8500 \mathrm{Oe}$, ce qui

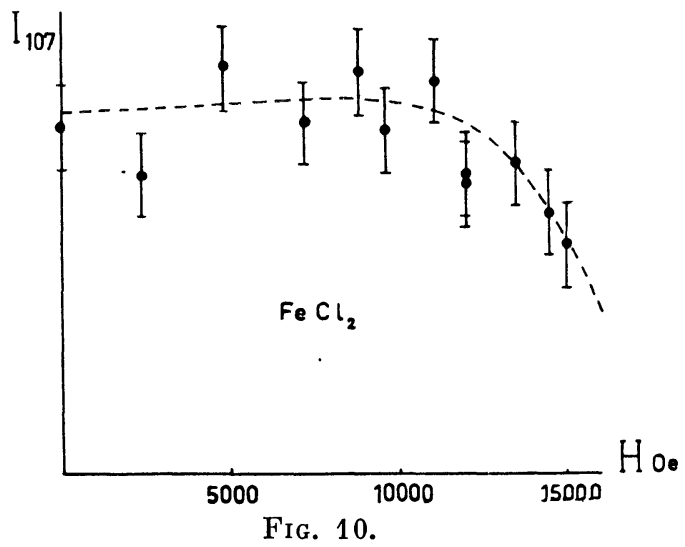

- est en assez bon accord, vu la précision des mesures, avec les résultats des mesures magnétiques de Starr Bitter et Kaufmann qui donnent 10000 Oe comme ordre de grandeur de ce champ.

4. Diffusion critique au voisinage du point de Curie. - Dans la structure magnétique que nous venons d'étudier, l'arrangement des moments magnétiques était bien ordonné. Nous allons maintenant envisager le.cas d'une structure magnétique partiellement désordonnée, c'est-à-dire dans laquelle il y a de fortes fluctuations dans l'orientation des moments magnétiques. De telles fluctuations d'aimantation existent dans un ferromagnétique lorsque la température est voisine de la température de Curie [7]. Ceci se traduit expérimentalement par une diffusion magnétique anormale qui n'est pas sans analogie avec la diffusion de la lumière par les fluides au voisinage du point critique. La figure 11 représente la variation de l'intensité diffusée à $3^{\circ}$ en fonction de la température dans le cas du fer, au voisinage du point de Curie. Nous avons choisi d'étudier le fer parce que l'intensité diffusée, proportionnelle au carré du moment magnétique, est beaucoup plus forte que dans le cas du nickel. Le but des expériences est de déterminer les paramètres au moyen desquels on peut donner une description des fluctuations. Ces fluctuations peuvent se décrire en termes de corrélations entre les moments d'atomes situés à la distance R. Soit

$$
\gamma(\mathbf{R})=\frac{1}{M^{2}}<\mathbf{M}(0) . \mathbf{M}(\mathbf{R})>_{\text {moy. }}
$$

la fonction de corrélation correspondante. A très basse température, tous les moments magnétiques sont parallèles, et la fonction de corrélation est égale à 1. A très haute température, dans le domaine paramagnétique, les moments magnétiques sont indépendants, et la fonction de corrélation est nulle, quel que soit $R$. A une température intermédiaire, c'est une fonction de $R$ présentant un profil en "toile de tente " tel que le représente la figure 12. On peut la décrire grossièrement par la portée de corrélations $1 / x$. Si la distance de deux

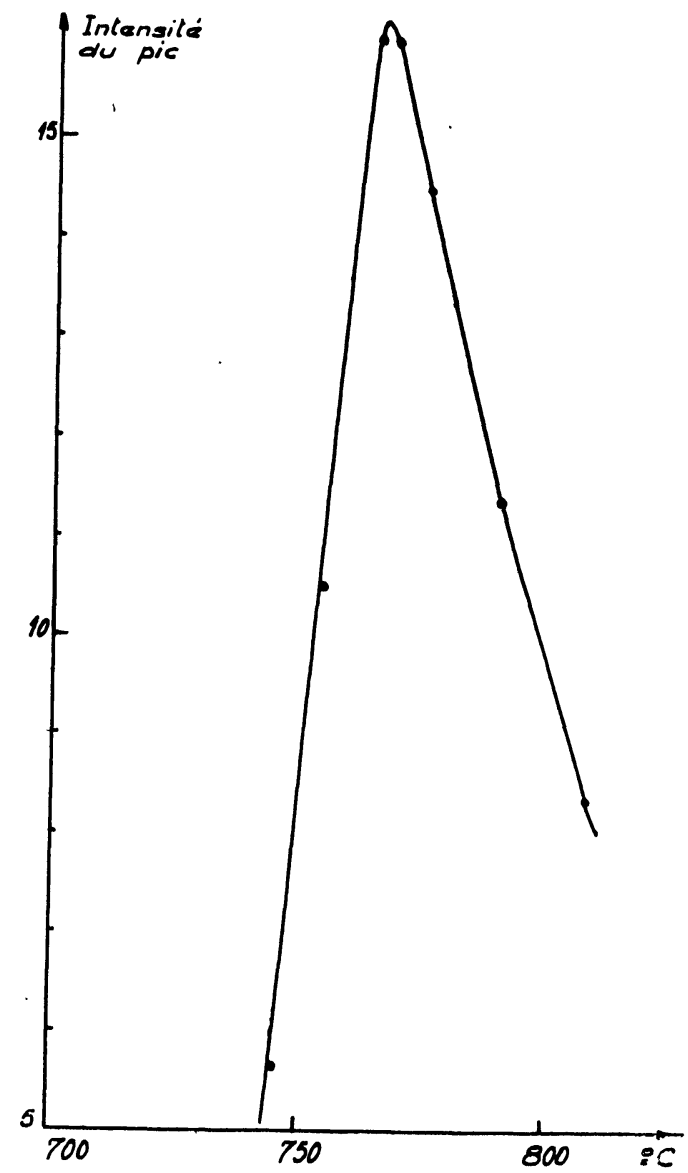

FIG. 11.

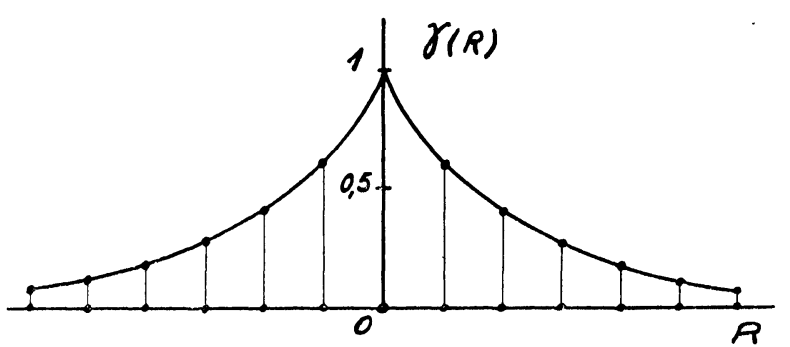

FIG. 12

atomes est supérieure à $1 / x$, leurs moments magnétiques sont indépendants, donc $\gamma=0$. Si leur distance est inférieure à $1 / x$, la corrélation est différente de zéro. Notons que pour $R=0, \gamma=1$, par définition de $\gamma(\mathbf{R})$. On peut montrer qu'asymptotiquement la fonction a pour expression

$$
\gamma(\mathbf{R})=C \frac{\mathrm{e}^{-x R}}{-R} .
$$


Calculons le carré de la fluctuation d'aimantation. On a

$$
\begin{aligned}
& \left\langle\left|\underset{\mathbf{R}}{\sum} \mathbf{M}(\mathbf{R})\right|^{2}\right\rangle=\underset{\mathbf{R}}{\sum} \sum_{\mathbf{R}^{\prime}}\left\langle\mathbf{M}(\mathbf{R}) \mathbf{M}\left(\mathbf{R}^{\prime}\right)\right\rangle \\
& \quad=N \sum_{\mathbf{R}^{\prime \prime}}\left\langle\mathbf{M}(\mathbf{R}) \cdot \mathbf{M}\left(\mathbf{R}+\mathbf{R}^{\prime \prime}\right)\right\rangle=N . M^{2}{\underset{\mathbf{R}}{2} \gamma(\mathbf{R}) .}
\end{aligned}
$$

Donc l'aire délimitée par la courbe $\gamma(R)$ est proportionnelle au carré de la fluctuation d'aimantation. Cette fluctuation devenant très grande au voisinage du point de Curie, l'aire délimitée $\operatorname{par} \gamma(R)$ devient très grande. Comme $\gamma(0)=1$, on en déduit

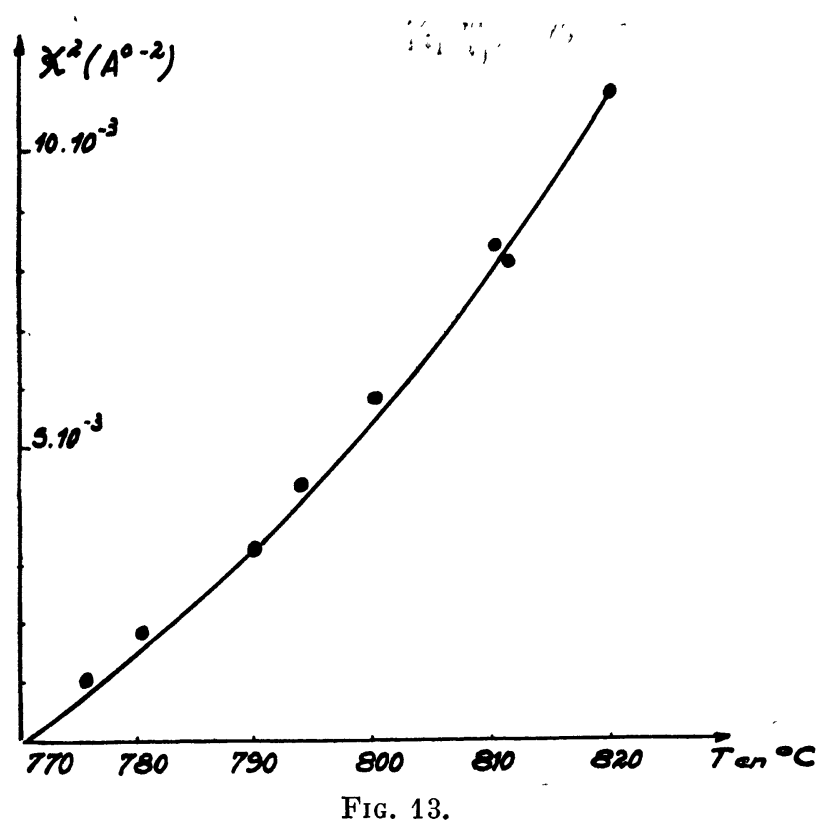

que les ailes deviennent très grandes, donc que $x$ devient très petit. Un calcul plus précis montre que

$$
\varkappa^{2}(T)=\frac{12}{a^{2}} \frac{\left|T-T_{\mathrm{c}}\right|}{T_{\mathrm{c}}} .
$$

où $a$ est la distance entre moments magnétiques. L'intensité magnétique diffusée dans une direction repérée par le vecteur de diffusion $\mathbf{K}$ est la transformée de Fourier de $\gamma(\mathbf{R})$ :

$$
I(\mathbf{K})=\sum_{\mathbf{R}} \mathrm{e}^{i \mathbf{K} \cdot \mathbf{R}} \gamma(\mathbf{R}) .
$$

A basse température, $\gamma(R)=1$. L'intensité est donc localisée sur des pics de réflexion sélectives ( $\mathbf{K}$ doit être égal à un vecteur du réseau réciproque $\tau)$. A très haute température, $\gamma=0$, il n'y a pas de diffusion magnétique. A une température intermédiaire, on trouve en utilisant la forme asymptotique (7) :

$$
I(\mathbf{K}) \sim \frac{1}{(\mathbf{K}-\tau)+x^{2}}
$$

Nous avions étudié la diffusion en avant $(\tau=0)$, le faisceau diffusé faisant un angle de $3^{\circ}$ avec le faisceau incident. De la mesure de l'intensité diffusée, on peut déduire facilement la valeur de $x$. La figure 13 représente la variation de $x$ avec la température. Ce résultat est en bon accord avec l'expression théorique de $x$ donnée plus haut.

L'expérience ayant été faite avec un spectromètre à temps de vol, on peut également déterminer la dynamique des fluctuations. En effet, de même que $I(\mathbf{K})$ est la transformée de Fourier de $\gamma(r)$, la section efficace différentielle est la transformée de Fourier de la fonction de corrélation spatiotemporelle :

$$
I(\mathbf{K}, \omega) \sim \int \mathrm{d} t \sum_{\mathbf{R}} \gamma(\mathbf{R}, t) \mathrm{e}^{i(\omega t+\mathbf{K} \cdot \mathbf{R})}
$$

où $\hbar \omega$ est la variation d'énergie du neutron et

$$
\gamma(\mathbf{R}, t)=\frac{1}{M^{2}}<\mathbf{M}(0,0) . \mathbf{M}(\mathbf{R}, t)>.
$$

Les fluctuations sont un phénomène irréversible, et il est raisonnable d'admettre que leur comportement est régi par une équation du type équation de diffusion :

$$
\frac{\partial \gamma(\mathbf{R}, t)}{\partial t}=\Lambda . \Delta \mathbf{R} \gamma(\mathbf{R}, t)
$$

$\Lambda$ est un paramètre phénoménologique qui décrit le comportement dans le temps 'des fluctuations. D'une façon plus précise $\Lambda x^{2}$ est l'inverse d'un temps de relaxation $\tau$ qui est relié à la variation moyenne d'énergie par $\hbar \bar{\omega}=\hbar / \tau=\hbar \Lambda x^{2}$. L'équation (11) permet de déterminer la transformée de Fourier de $\gamma(\mathbf{R}, t)$, donc l'intensité diffusée. En pratique, la variation d'énergie est beaucoup plus petite que la largeur de la raie incidente. Il faut donc calculer la forme de la raie diffusée pour différentes valeurs de $\Lambda$, ce qui permet de déterminer la valeur de $\Lambda$ qui cadre le mieux avec le résultat expérimental. Les premières mesures de $\Lambda$ ainsi faites n'ont pu nous donner qu'un ordre de grandeur. Cependant les résultats semblent en meilleur accord avec les calculs faits en utilisant un modèle de Heisenberg pour le fer plutôt qu'avec ceux qui utilisent un modèle de bandes.

Manuscrit reçu le 8 février 1958.

\section{BIBLIOGRAPHIE}

[1] Breton, Hubert, Meriel, J. Physique Rad., 1957, $18,25$.

[2] Brockhouse (B. N.), Canad. J. Phys., 1955, 33, 889.

[3] Galula, Jagrot et Netter, J. Physique Rad., 1954 15, $59 \mathrm{~S}$; JÁcrot et Gobert, J. Physique Rad., 1958, 19,82 et $1958,19,51 \mathrm{~A}$.
[4] Starr, Bitter et Kaufman, Phys. Rev., 1940, 58, 977.

[5] Wilkinson (M. K.) et Gable (J. W.), Bull. Amer. Phys Soc., (2), 1956, 1, n $4,190$.

[6] Néel (L.), Xe Congrès Solvay, 1954, p. 251 ; Stoops Bruxelles, 1955.

[7] Van Hove (L.), Phys. Rev., 1954, 95, 1374. 